\section{Automated S/TEM Sample Preparation for Semiconductor Process Support}

\author{
Greg Cuti* and Taha Jabbar** \\ ${ }^{*}$ Sela USA, Inc. Sunnyvale, CA, USA \\ and ${ }^{* *}$ Athenian Institute, Danville, CA, USA \\ e-mail contact: greg@sela.com
}

Smaller devices drive demand for high resolution imaging

The semiconductor industry has unquestionably entered the realm of nanotechnology. Critical dimensions of many features are specified in nanometers. Gate oxides are only a few nanometers thick. Barrier and seed layers for copper processes are not much more. Gate lengths are forecast at less than $20 \mathrm{~nm}$ by the end of the decade. Additionally, the drive to increase device density is leading to the adoption of FinFET and other new transistor designs that include complex three-dimensional structure. Even conventional planar CMOS designs now incorporate processes such as damascene interconnects that are inherently three dimensional. The combined need for higher spatial resolution and cross-sectional imaging of complex structures has led to a significant increase in the demand for scanning transmission electron microscopy (STEM) and (TEM) in semiconductor manufacturing operations.

Both TEM and STEM require very thin samples, typically less than $100 \mathrm{~nm}$ thick, that can be difficult, time consuming, and expensive to prepare. Sample preparation can become a process bottleneck. Manual sample preparation is slow and requires a skilled technician. Focused ion beam (FIB) preparation provides an alternative that is fast but expensive. Automated pre-thinning systems, such as the EM2, (SELA) perform the preliminary stages of the thinning quickly, reliably, and at a fraction of the cost of using FIB for the entire sample extraction and thinning process.

The primary limitation to SEM resolution in most applications is beam spreading within the bulk specimen. Beam electrons scatter as they enter the specimen, eventually giving up all of their energy through multiple scattering events, unless one of those events directs the beam electron back out through the sample surface as a backscattered electron (BSE). The region that encompasses these scattering events is known as the volume of interaction and the imaging signals used by SEMs can originate anywhere within this volume. It is typically many times larger than the beam diameter though it can be reduced by operating at low accelerating voltages.

Both TEM and STEM form images from electrons transmitted through the sample and thus require sample thin enough to transmit most of the beam electrons. In a STEM, like the SEM, the beam is focused to a small spot that scans the sample surface. Since the sample is thin, most electrons scatter once or not at all as they pass through. The volume of interaction is greatly reduced and confined almost entirely to the region directly below the beam spot. In a sufficiently thin sample, STEM resolution is determined by the size of the beam.

A TEM uses a relatively broad electron beam and focuses transmitted electrons into a real image that is projected onto a fluorescent screen or some other imaging device. Its resolution is determined primarily by the optical performance of its electron lenses. However, like STEM, sample thickness (or rather thinness) is a critical determinant of imaging performance and thinner is almost always better.

\section{Sample Preparation}

Historically, S/TEM's extensive sample preparation requirements impeded its acceptance in applications where high throughput and rapid results were important. Manual preparation techniques were difficult, time consuming, and unreliable. The recent development of FIB based sample preparation techniques has been a significant factor in the growing acceptance of S/TEM in semiconductor manufacturing.
The primary benefits of FIB based preparation are its ability to reliably create site specific sections of designated devices or defects, and its precise control of the final thinning process. However, FIB systems are expensive and because of their versatility they are often among the most heavily used tools in the fab support laboratory. In many cases it is more economical to consider other methods for the initial stages of the preparation process, saving expensive FIB time for the final thinning process where its precise control adds the greatest value and provides the highest return on investment. We describe below three methods for the initial phase of sample preparation: manual prethinning, FIB lift-out, and automated pre-thinning. All three create a thick section ready for final thinning to electron transparency.
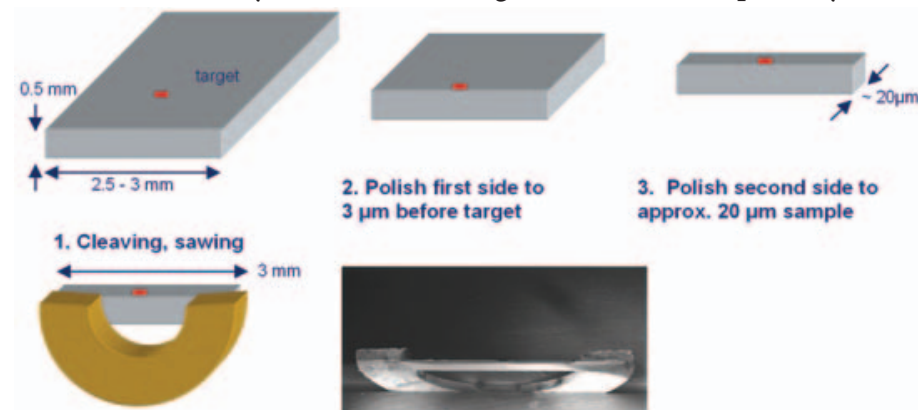
$3 \mu \mathrm{m}$ before target

3. Polish second side to approx. 20 um sample

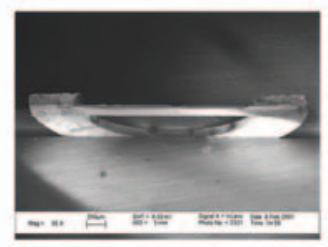
4. Gluing to TEM sample holder

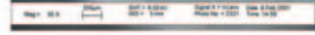
SEM image of man
prepared sample

Figure1 Manual pre-thinning sample preparation. All steps are manual, facilitated by specially designed, in-house jigs and holders. FIB ready samples are available in about 4 hours.

\section{Manual Pre-thinning}

Figure 1 illustrates a typical manual pre-thinning process flow:

1) Cleave or cut (tungsten wire saw) the wafer to obtain a piece 2.5 $\mathrm{mm}$ to $3 \mathrm{~mm}$ in width and containing the target near an edge.

2) Polish the edge with diamond films of diminishing grain size $(15 \mu \mathrm{m}, 9 \mu \mathrm{m}, 3 \mu \mathrm{m}, 1 \mu \mathrm{m})$ to within approximately $3 \mu \mathrm{m}$ of the target

3) With tungsten wire saw, cut section approximately $300 \mu \mathrm{m}$ thick containing the target.

4) Polish to approximately $20 \mu \mathrm{m}$ thickness using diamond films and in house polishing jig.

5) Glue a half-ring TEM sample grid to the $20 \mu \mathrm{m}$ Si lamella.

6) Cure glue on hot plate $\left(120^{\circ} \mathrm{C}\right.$ for 20 minutes $)$ and remove from holder.

7) FIB mill to final thickness.

Manual preparation is relatively slow, typically requiring about 4 hours to prepare a FIB-ready sample. It requires very little capital investment, but this cost savings is offset somewhat by the labor expense for a skilled technician.

\section{FIB Lift-out}

In the "lift-out" process, the FIB first cuts a relatively thick section from the full wafer. The section is attached to a probe needle of a micromanipulator, then transported and attached to a TEM grid inside the FIB chamber using the FIB cutting and metal deposition capabilities. The entire process takes abut half an hour. There is some opportunity to reduce this time through automation though the bulk of it is consumed by milling and deposition processes that cannot be made significantly faster. FIB time is expensive. FIB systems require significant initial investment (1-2 M\$) and ongoing support costs.

\section{Automated Pre-thinning}

The automated pre-thinning process prepares mounted, sitespecific thick-sections ready for final FIB thinning. It can create both cross-sectional and plan-view samples. Cutting is accomplished with dry, cryo-cooled diamond sawing that is independent of the crystal 


\section{thinkforward}

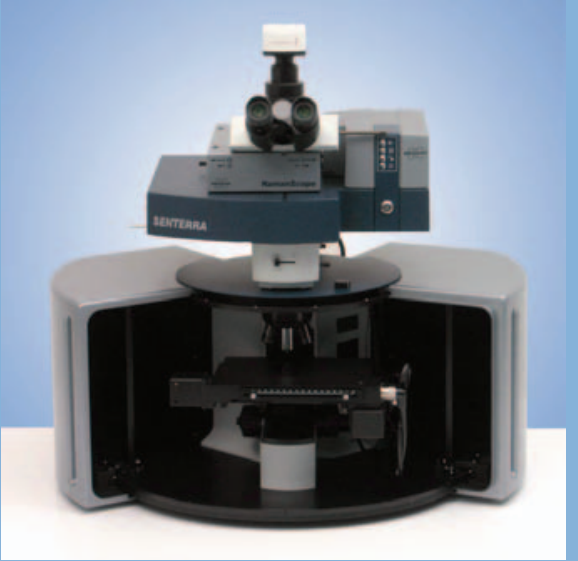

$\square$ All-in-one, compact, confocal design

$\square$ Multiple wavelegths; $1064 \mathrm{~nm}$, $785 \mathrm{~nm}, 532 \mathrm{~nm}$ and/or $633 \mathrm{~nm}$.

$\square$ Sure_Cal ${ }^{\oplus}$ automatic continuous calibration

$\square$ Spectral imaging utilizing sample stage mapping

$\square$ Confocal depth profiling with FlexFocus $^{\mathrm{TM}}$

$\square$ Automatic fluorescence rejection using SERDS for $785 \mathrm{~nm}$

\section{Isn't it time to make peace between two technologies?}

\section{Introducing the SENTERRA ${ }^{\text {TM }}$}

Dispersive Raman Microscope with the new integrated 1064nm FT-Raman technology

Bruker Optics introduces the first Raman microscope system to combine the long wavelength benefits of $1064 \mathrm{~nm}$ excitation with the scattering benefits of shorter wavelength Raman excitation. The new 'hybrid' platform accommodates the RamanScope fluorescence-free Fourier transform Raman system and the SENTERRA grating based dispersive Raman technology with fluorescence rejection tools. This combination provides full spectroscopic characterization and optimizes the strengths of the both techniques for your complex micro-analysis samples.
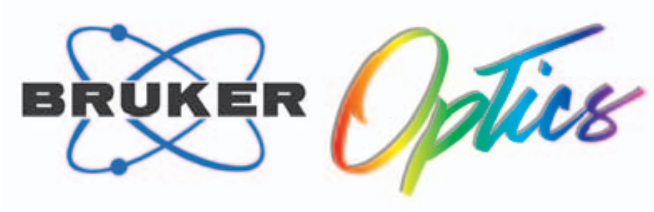

for more information please visit:

www.brukeroptics.com/microscopy 1-888-4BRUKER | microscopy@brukeroptics.com 


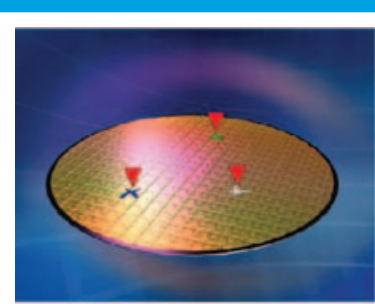

1. Mark Targets

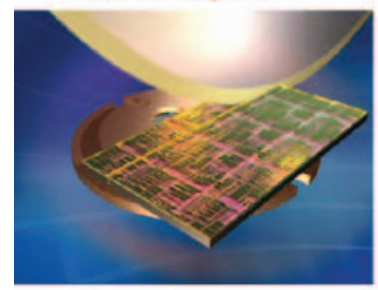

4. Initial Separation

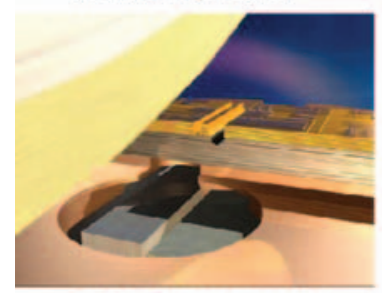

7. Trench (no contact with target sidewall)

Figure 2. Automated pre-thinning sample preparation with SELA EM2. Except for step 2 , the entire process is fully automated and proceeds without operator intervention. FIB ready samples are available in 25-35 minutes.

orientation of the underlying wafer, allowing it to accommodate angled pattern alignments and non-crystalline substrates. The system uses an automated stage and a high resolution optical microscope for target identification. It accepts up to $300 \mathrm{~mm}$ wafers and can navigate automatically to predefined target coordinates. In the finished sample the target is located within a ridge $10-20 \mu \mathrm{m}$ thick and approximately $100 \mu \mathrm{m}$ tall, supported on a somewhat thicker base section that is glued to a TEM compatible grid.

Mechanical cutting and polishing procedures, whether manually and automatically controlled, risk damaging the sample through chipping, cracking or delamination. The automated system described here reduces this risk by eliminating wet polishing, and by using a special "detachment" procedure to isolate the target region from its surroundings prior to sawing. Once targets have been designated the entire process proceeds with minimal need for operator intervention.

Automated pre-thinning process flow (figure 2):

1) Navigate to the target on a full wafer interactively or using coordinates provided by upstream inspection.

2) Designate target(s) with integrated marking system (ink).

3) Plan and execute sawing or cleaving operations (externally) to reduce wafer to smaller sections (up to $18 \mathrm{~mm}$ x $18 \mathrm{~mm}$ ) containing the target(s).

4) Return section for automated sawing and mounting to create a final FIB-ready sample.

Automated pre-thinning sample preparation is fast (25 - $35 \mathrm{~min}$ utes), reliable, and relatively inexpensive. Initial capital cost $(\sim 0.30$ $\mathrm{M} \$$ ) is $5 \mathrm{X}$ less than FIB, and ongoing maintenance costs are commensurately lower. Operator labor expense is also reduced, requiring lower skill levels and less time than manual pre-thinning.

\section{Discussion}

In a typical lab, each of the three pre-thinning techniques will be the best choice in some circumstance. In labs where automated pre-thinning is available it becomes the method of choice for most routine analyses, and the other approaches are used on an exceptional basis. Lift-out is slightly faster on single samples, while automated pre-thinning is faster on multiple samples. Lift-out is expensive. The difference in speed is more than offset by the high cost of FIB time: the amortization expense alone is at least five times greater than automated pre-thinning. Off-loading the preliminary phases of sample preparation to the automated pre-thinning system frees up the FIB for other more valuable uses. In practice automated prethinning is the best choice for routine jobs including two or more samples, with lift-out reserved for special circumstances, such as when preparing one sample per job, when the sample size is very small, or when the chip is bonded to a package. In addition to the general considerations of economy and efficiency, automated pre-thinning has capabilities that make it the preferred method in certain circumstances. For example, automated pre-thinning can create plan view samples as well as cross-sections. It readily accommodates large targets that are slow and difficult for lift-out - such as cross sections through arrays of contacts or other extended features. Finally, unlike polishing, automated pre-thinning conserves the unsampled portions of the wafer, making them available for rework or resampling should the initial attempt prove unsuccessful.

\section{Conclusion}

The need for high-resolution imaging and analysis to support semiconductor manufacturing is increasing the demand for S/TEM capability. Preparation of the ultra-thin samples required for these techniques introduces significant cost and delay into the analytical process. Manual techniques are slow, and their low initial investment is offset by higher ongoing labor expenses. FIB lift-out is expensive. Automated pre-thinning sample preparation is less expensive than lift-out and faster for multiple sample jobs. By freeing the FIB to perform tasks for which it is uniquely capable, automated pre-thinning optimizes the allocation of resources in the analytical laboratory. Higher throughput and reduced costs provide a direct benefit in improved laboratory productivity, as illustrated in figure 3.

\section{Acknowledgements:}

Taha Jabbar was employed as a summer intern at SELA and provided valuable assistance with literature and lab research as well as writing the initial draft of this article.

\section{Manual Pre-thinning}

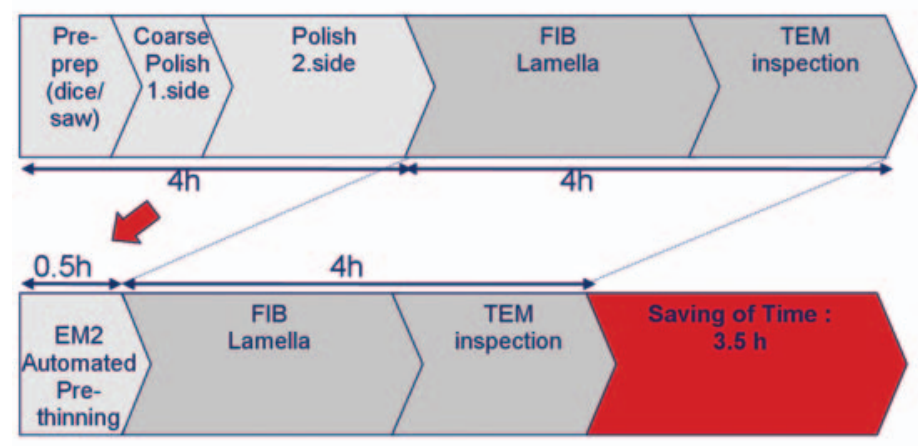

\section{Automated Pre-thinning}

Figure 3. Comparison of time and process flow for manual and automated sample preparation. The net time savings of 3.5 hours is a reduction of nearly $50 \%$ in time-to-result. 
Why have 1,000 scientists in 25 countries selected Minus $\mathbf{K}^{\circ}$ vibration isolators?

Because Minus K vibration isolation systems deliver $10 x$ to $100 x$ better performance than high-performance air systems, without air, and for air table prices.

\section{Visit www.minusk.com for more information..}

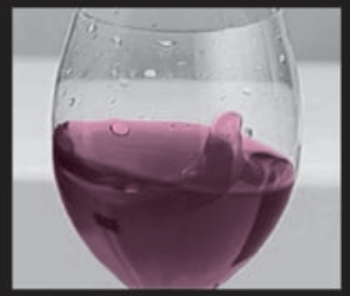

Without Minus $\mathbf{K}$

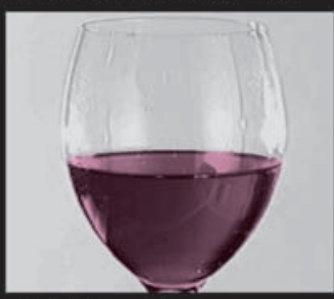

With Minus K

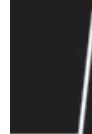

RATION ISOLATION BY:

minus $\mathbf{k}^{\odot}$ TECHNOLOGY

420 S. Hindry Ave., Unit E • Inglewood, CA 90301 USA Phone: 310-348-9656 - Fax: 310-348-9638

sales@minusk.com•www.minusk.com

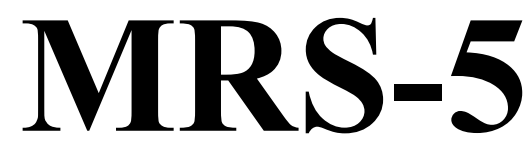

We are ISO-9000 certified and ISO-17025 accredited Microscopy Calibration Standard Now you can calibrate from 1,000X to $1,000,000 X !$

This is our fourth generation, traceable, magnification reference standard for all types (SEM, FESEM, Optical, STM, AFM, etc.) of microscopy. The MRS-5 has multiple $\mathrm{X}$ and $\mathrm{Y}$ pitch patterns ranging from $80 \mathrm{~nm}( \pm 1 \mathrm{~nm})$ to $2 \mu \mathrm{m}$ and $3 \mathrm{bar}$ targets from $80 \mathrm{~nm}$ to $3 \mu \mathrm{m}$. There is also a STM test pattern.

Free web resource guide!

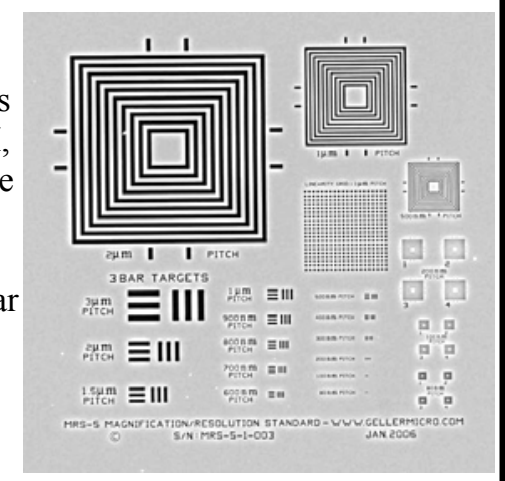

GELLER MICROANALYTICAL LABORATORY, Inc.

426e Boston St., Topsfield, Ma 01983 www.gellermicro.com

\section{SII $0^{\circ}$ \\ “SCIENTISTS EVERYWHERE ARE BEAMING"}

SII NanoTechnology USA Inc.

Silicon Multi-Cathode X-Ray Detector

NO LN2 • Active area $\sim 50 \mathrm{~mm}^{2} \bullet<133 \mathrm{eV}$ FWHM at $5.9 \mathrm{keV} \cdot$ ICR $1.5 \mathrm{Mcps} \cdot \mathrm{OCR}$ up to $600 \mathrm{kcps}$

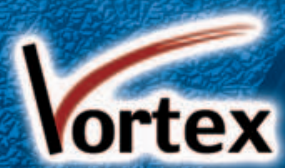

EDS - Fast Mapping - SEM - Microanalysis - Process Control 